\title{
UV-decontamination of potable and sewage water in the city with population over one million
}

\author{
Aleksandr Smirnov ${ }^{1 *}$, Evgeniy Pupyrev ${ }^{2}$, Ilkhomzhon Shukurov $^{2}$ and Konstantin Chertes ${ }^{3}$ \\ ${ }^{1}$ AO “NIIVODGEO”, 19435, B. Savvinsky per., 9, p. 1., Moscow, Russia \\ ${ }^{2}$ Moscow State University of Civil Engineering, 129337, Yaroslavskoye Shosse 26, Moscow, Russia \\ ${ }^{3}$ Samara State Technical University, 443100, 244 Molodogvardeyskaya st., Samara, Russia
}

\begin{abstract}
The waterworks system in a modern city is a complex challenge. From the one hand, it is necessary to provide high-quality potable water to the residents with observance of all sanitary and hygienic requirements; from the other hand, the sewage water discharged from the city should not affect the environment. Meanwhile, the microbiological safety is the top-priority and crucial parameter for evaluation of any work and any project. In Novosibirsk, solutions have been found for both of them by using the cutting-edge approaches in the decontamination technologies. The UV-decontamination enabled to create a multi-barrier efficient protection when dealing with the potable water treatment and ensure environmentally-friendly decontamination of the sewage water.
\end{abstract}

The location of Novosibirsk near one of the biggest rivers in Russia, the Ob river, imposes certain duties concerning the use of water in the city. $\mathrm{Ob}$ is the source of centralized potable water supply in Novosibirsk, and the sewage water discharged into this river may significantly affect the ecological status of the river downstream.

In order to solve these problems, several modern technological solutions have been consistently applied; these solutions ensured the observance of all microbiological requirements for the potable water and sewage water.

Novosibirsk is the largest city in the Russian Federation which has implemented environment-friendly and safe method of UV-decontamination in the municipal water treatment plants and sewage treatment plants.

\section{Potable water supply}

During the course of the 'Water supply and water disposal systems development in 20072013' program implementation, in 2011, a municipal water UV-decontamination plant has been launched at the Pumping \& Filtration Station No. 1 (NFS-1) with the rated capacity amounting to 350 ths. $\mathrm{m}^{3} /$ day. According to the expert opinion issued by 'Novosibirsk Oblast Hygiene and Epidemiology Center' Federal State Healthcare Institution in 2008, the

\footnotetext{
*Corresponding author5171434@mail.ru
} 
river water is affiliated to the 2-nd class in terms of microbiological contamination. Therefore, in order to create an efficient multi-barrier technology, the UV-radiation decontamination and chlorination (chlorine-ammonia) has been used in order to provide long-lasting disinfecting effect.

In two years, the next stage of the city water treatment system modernization has been implemented: UV-decontamination plant has been launched at the Pump \& Filtration Station No. 5 (NFS-5). The rated capacity of this plant amounts to 500 ths. $\mathrm{m}^{3} /$ day. At this station, the multi-barrier decontamination system has also been implemented.

The multi-barrier decontamination technology that combines the UV-radiation and chlorination enables to use the advantages of each of the methods. UV-radiation is characterized by maximum decontaminating efficiency even when dealing with the microorganisms resistant to conventional chlorination (such as viruses, and protozoa cysts). Chlorination allows to ensure the long-lasting decontamination effect, which preserves the quality of water inside the distribution grids. Reduction of the actually used chlorine doses is beneficial for the reduction of the organochlorine compounds concentrations in the potable water [7].

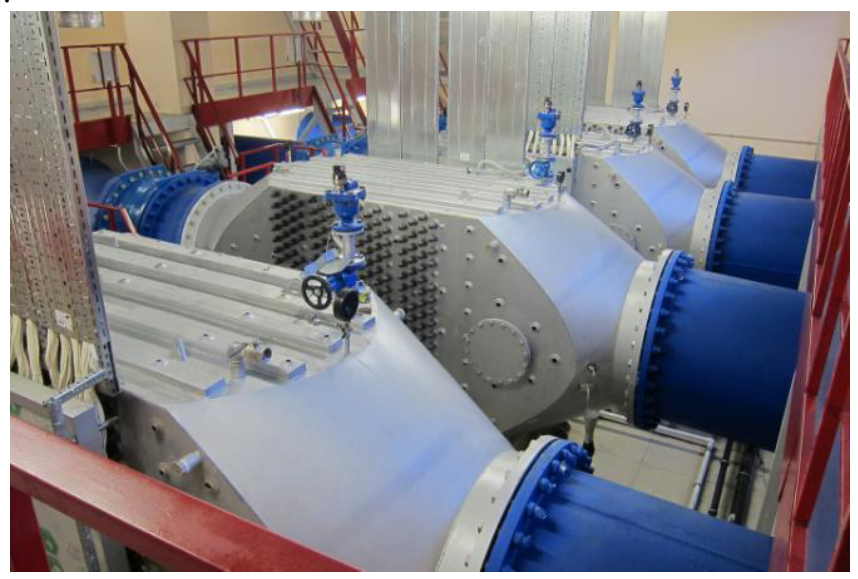

Fig. 1. UV-equipment at NFS-5

\section{Sewage water treatment}

The observance of the state requirements for the sewage water treatment (SanPiN 2.1.5.980-00 'Hygienic requirements for the surface water protection') implies the mandatory decontamination of the city treated sewage water in order to prevent the ingress of the potentially pathogenic microorganisms into the environment and water bodies that may be later used as the potable water sources [4].

The desired degree of the potable water decontamination may be ensured by multiple methods; the most widely used one is the reagent-based treatment (chlorination), and the use of UV-radiation[1-3].

The use of chlorine or other chlorine-containing reagent (hypochlorite) observing the recommended concentrations for ensuring protection against the bacterial contamination does not guarantee the protection against the viruses and other chlorine-resistant microorganisms (such as parasitic protozoa). The ingress of the residual chlorine into the water body - sewage water receiver - may significantly deteriorate the ecological status of such water body up to complete seizure of the self-purification processes and death of all life forms. The sewage water chlorination is also accompanied by the active formation of by-products (various organochlorine compounds) that may be characterized by high 
toxicity, mutagenic action, and cancerogenic action. Even one-time contamination with such products may lead to long-lasting harm to the water body biocoenosis.

Modern applicable Russian requirements for the sewage treatment facilities (SP 32.13330.2012 'Updated version of SNiP 2.04.03-85 'Sewage. External grids and facilities") require the mandatory stage of dechlorination after the decontamination accomplished with chlorine or other chlorine-containing reagent. Dechlorination allows to remove the residual chlorine, but cannot prevent the formation and ingress of the organochlorine compounds into the water body, which have been formed during the chlorination process [6].

Decontamination accomplished using the UV-radiation is characterized by a number of rock-solid advantages:

- $\quad$ High efficiency with regard to all types of microorganisms (bacteria, viruses, and protozoa), even in their resistant cyst forms;

- The water temperature and pH-factor do not affect the UV-decontamination efficiency;

- Absence of consequences excludes the adverse effect for the water body and environment;

- No decontamination by-products;

- Easy control and management of the decontamination process; automation is possible;

- The method is safe for the public, there is no need to arrange storage facilities with toxic chlorine-containing reagents that require observance of the dedicated process safety and environmental safety measures, which improves the reliability of the sewage systems in general;

- $\quad$ High energy efficiency;

- UV-radiation decontamination leads to lower operational costs in comparison with the chlorination-dechlorination, especially when using safer, but more expensive sodium hypochlorite.

For efficient and complete fulfillment of the requirements concerning the sewage water microbiological quality parameters of the sewage water discharged into the water body, the UV-decontamination technology has been adopted, which is based on the amalgam ozonefree low-pressure lamps.

In February 2016, the Sewage Water UV-Decontamination Station was put into operation at the Municipal Sewage Water Treatment 1-st Train, Novosibirsk, with the nominal flow rate amounting to $26,000 \mathrm{~m}^{3} / \mathrm{h}\left(480,000 \mathrm{~m}^{3} /\right.$ day $)$.

The domestic UV-equipment (NPO 'LIT') allows to efficiently decontaminate the sewage water, the UV-radiation dose in accordance with MU 2.1.5.732-99 and MUK 4.3.2030-05 amounts to at least $30 \mathrm{~mJ} / \mathrm{cm}^{2}$.

The UV-station comprises three working channels. Each channel includes three working sections with two 88MLV UV-modules in each of them. Further, channels comprise one redundant section in each of them; these redundant sections are to be used during the routine maintenance. The power consumption of the entire UV-station amounts to as much as $495 \mathrm{~kW}$.

In order to bring down the sewage water UV-decontamination station footprint, this station is located within the running contact tank. The well-established two-level configuration has been used for the UV-station: the modules with the UV-lamps are located on the lower level in the concrete channels of the former contact tank, and all cabinets containing the UV-lamps control and power systems are located above the modules in a new easily-erected structure building. The entire UV-station is located inside the building $14 \times 13.5 \mathrm{~m}$. 
Vertical orientation of the UV-lamps enabled to reduce the area occupied by the UVstation and minimize the scope of service work, since there is no need to remove the module from the channel for the scheduled maintenance procedures (UV-lamps replacement). Modern high-performance amalgam low-pressure lamps are characterized by high efficiency and good service life (over 12,000 hours).

An important feature of such station is the UV-lamps radiation power regulation integrated system. Such systems enabling the significant savings of the consumed electric power depending on the quality and quantity of the sewage water being treated are rather new within the domestic practical applications. One of the features of the system used is that the power regulation or adjustment occurs not only depending on the sewage water flow rate, but also based on the integral value of the sewage water quality - sewage water transmission coefficient with regard to the UV-rays. In order to measure this coefficient, the station is equipped with an additional device - taumeter; it measures the transmission coefficient in real-time and automatic manner with no lag, and with no need to engage service personnel. Such a system used for the UV-lamps power adjustment enables to reduce the electric power consumption by as much as $30-40 \%$ of the UV-station power rating.

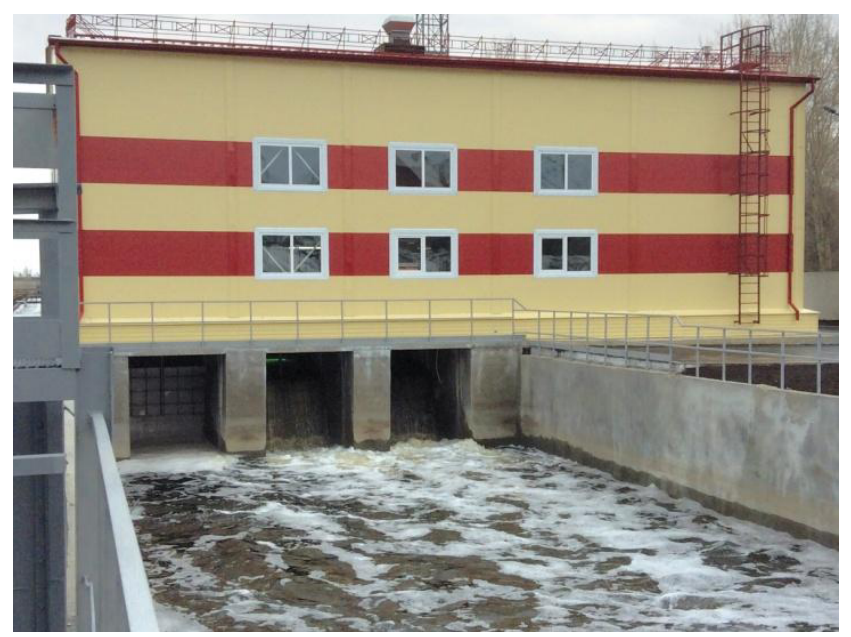

Fig.2. UV-station building, Novosibirsk

The UV-decontamination allowed to ensure efficient observance of the sanitary \& epidemiological and environmental safety of one of the most important water supply source in the region - the $\mathrm{Ob}$ river.

It should be noted that the UV-decontamination station complies with the most up-todate requirements concerning the use of the best available technologies (BAT) as stated in the Informational and Technical Manual ITS 10-2015 'Sewage water treatment using the centralized water disposal systems in settlements and urban districts'. This Manual specified that the use of chlorination, even with the follow-up dechlorination, is not the best available technology for the decontamination applied in the facilities related to such scale ('major' according to the classification provided in the Manual) [5]. 




Fig.3. Redundant UV-module

\section{Conclusion}

The biggest Siberian water service (Novosibirsk water service is in the 3-rd place in production capacity after Moscow and Saint Petersburg) has completely been transferred to the UV-decontamination, used both for potable water, and sewage water.

The potable water undergoes the treatment using the multi-barrier decontamination technologies (combination of UV andchlorine-ammonization), which enables to provide safe and tasty water to every citizen living in the city.

The UV-decontamination station built on the municipal sewage water treatment facilities is a reliable barrier that prevents the proliferation of pathogenic microorganisms and viruses, while causing no harm to the water bodies and environment.

The complex use of the modern UV-decontamination technology both in potable water treatment, and in sewage water treatment enabled to fulfill the most strict requirements of both the sanitary, and environmental legislation.

\section{References}

1. O.A. Guseva, M.M. Gerasimov, A.D. Smirnov, Water Supply and San. Eq., 2, 59-62 (2010)

2. L.M. Vasilyak, A.D. Smirnov, Water Supply and San. Eq., 9, 49-56 (2014)

3. S.Yu. Shishov, E.A. Troshkova, V.Ya. Zhukova, A.D. Smirnov, R.A. Davlyaterova, V.A. Smagin, Water Supply and San. Eq., 6, 32-38 (2014)

4. N. Zaletova, S. Zaletov, Int. Jour. of App. Eng. Res., 10, 43873-43874 (2015)

5. N. Zaletova, Y. Voronov, N. Makisha, Int. Jour. of App. Eng. Res., 10, 42454-42455 (2015)

6. E. Gogina, N. Makisha, App. Mech. and Mat., 587-589, 636-639 (2014)

7. V. Chukhin, A. Andrianov, V. Orlov, The steel pipe corrosion in drinking water distribution systems and its rehabilitation techniques (2014) International No-Dig Madrid 2014. 